\title{
ANALYTICAL INVESTIGATION OF A ONE-DIMENSIONAL HOMOGENIZED MODEL FOR A PRESSURIZED WATER REACTOR CORE
}

\author{
Joachim BENNER and Ulrich SCHUMANN \\ Kernforschungszentrum Karlsruhe GmbH, Institut für Reaksorentwicklung, Projekt Nukleare Sicherheit, Postfach 3640, D-7500 \\ Karlsruhe, Fed. Rep. Germany
}

Received 15 July 1981

\begin{abstract}
A one-dimensional homogenized model for dynamic fluid-structure interaction in a pressurized water reactor core is used to study the influence of the virtual density and spacer's stiffness. The model consists of a linear system of partial differential equations for fluid velocity, rod velocity and pressure. For these equations analytical solutions are deduced for boundary conditions prescribing either periodic wall oscillations or linearly growing wall accelerations from rest. The theoretical model for the virtual density is verified by comparison to an experiment. For zero spacer stiffness, purely acoustic oscillations appear. For positive spacer stiffness, additional oscillations arise with relative rod motions. The wavelengths of the latter oscillations are small for weak spacers. Large numerical effort would be required in a more complete three-dimensional core-model to resolve such short wave lengths. In fact in a typical core the spacer's stiffness $c_{\mathbf{s}}$ is small in comparison to the fluid bulk modulus $K$. For $c_{\mathrm{s}} / K<0.1$ it might be appropriate to neglect the influence of the spacers.
\end{abstract}

\section{Introduction}

In some recent papers [1-3] a three-dimensional model for a pressurized water reactor (PWR) core was developed. This model is designed for implementation in a code for the analysis of pressure loadings on vessel internal structures during a postulated loss-of-coolant accident. The model is given in terms of volume averaged fluid and rod velocities and the pressure. It includes several physical effects, so the relative motion of rods, rod bending and straining, forces due to elastical spacer springs and fluid friction. A summary of the basic features and the limitations of this 'homogenized' core model is given in [3].

Because of the various physical effects incorporated in this model and the dependence on three space dimensions its actual implementation is rather complicated. Therefore in [3] some simplifications were suggested. For example, it is rather obvious that in the first milliseconds of a blowdown accident, where the fluid velocity inside the core is small, fluid friction is less important. But it is by no means obvious whether the influence of the spacer springs is negligible too. This was suggested in [3] and will be further discussed here.

For this purpose we investigate a simplified one-dimensional core-model and derive analytical solutions for some special initial and boundary conditions. The analytical solution process and the resultant velocity-distributions under different stiffness parameters are discussed here. From these solutions, values for the dynamically effective core-mass and the effective speed of sound are derived.

Another aim of this paper is to compare some experimental results [4] with theoretical predictions of the homogenization theory. 


\section{One-dimensional, homogenized core-model}

The linear, one-dimensional model equations are:

$$
\begin{aligned}
& \alpha \rho_{0} \dot{U}+\rho_{0} \bar{\rho}(\dot{U}-\dot{V})=-\alpha \partial P / \partial x, \\
& (1-\alpha) \rho_{\mathrm{s}}^{0} \dot{V}+\rho_{0} \bar{\rho}(\dot{V}-\dot{U})=c_{\mathrm{s}} \frac{\partial^{2} W}{\partial x^{2}}-(1-\alpha) \frac{\partial P}{\partial x}, \\
& \frac{\dot{P}}{K}+\frac{\partial}{\partial x}(\alpha U+(1-\alpha) V)=0,
\end{aligned}
$$

with

$$
\begin{array}{lll}
\dot{U} \equiv \partial U / \partial t, & V \equiv \dot{W}, & U=U(x, t), \\
V=V(x, t), & P=P(x, t), &
\end{array}
$$

Here $U$ and $V$ denote fluid and structure velocity, $P$ the pressure. The meaning of the material constants can be found in table 1. Suitable initial- and boundary conditions are described in a following chapter. In order to reduce the number of unknowns and equations we differentiate (1) and (2) with respect to the time- and (3) with respect to the space variable. Then the pressure $P$ can be eliminated. In a next step we make the variables non-dimensional, using as characteristic scales:

length: $L, \quad$ time: $\tau=\left[\left(L^{2} \rho_{0}\right) / K\right]^{1 / 2}$.

Subsequently, we use non-dimensional quantities.

After some simple rearrangements of the coefficients this results in two coupled partial differential equations for fluid and structure velocity:

$$
\underline{A} \frac{\partial^{2}}{\partial t^{2}} \boldsymbol{C}(x, t)-\underline{B} \frac{\partial^{2}}{\partial x^{2}} \boldsymbol{C}(x, t)=0, \quad-1 \leqslant x \leqslant 1,
$$

with

\begin{tabular}{|c|c|c|}
\hline$\rho_{0}$ & $\left(\mathrm{~kg} / \mathrm{m}^{3}\right)$ & Fluid density \\
\hline$\rho_{\mathrm{s}}^{0}$ & $\left(\mathrm{~kg} / \mathrm{m}^{3}\right)$ & Rod density \\
\hline$a$ & $(\mathrm{~m} / \mathrm{s})$ & Sound-speed, fluid \\
\hline$a_{\mathrm{s}}$ & $(\mathrm{m} / \mathrm{s})$ & $\begin{array}{l}\text { Sound-speed, due to } \\
\text { rod compression }\end{array}$ \\
\hline$\alpha$ & $(-)$ & Fluid volume fraction \\
\hline$K$ & $\left(\mathrm{~N} / \mathrm{m}^{2}\right)$ & {$\left[\alpha /\left(\rho_{0} a^{2}\right)+(1-\alpha) /\left(\rho_{s}^{0} a_{s}^{2}\right)\right]^{-1}$} \\
\hline$c_{s}$ & $\left(\mathrm{~N} / \mathrm{m}^{2}\right)$ & $\begin{array}{l}\text { Stiffness per unit rod length } \\
\text { of a spacer spring }\end{array}$ \\
\hline $\bar{\rho}$ & $(-)$ & Virtual density coefficient \\
\hline
\end{tabular}

$$
\underline{A}=\left\{\begin{array}{ll}
\alpha+\bar{\rho} & -\bar{\rho} \\
-\bar{\rho} & (1-\alpha) \kappa+\bar{\rho}
\end{array}\right\}, \quad \underline{B}=\left\{\begin{array}{ll}
\alpha^{2} & \alpha(1-\alpha) \\
\alpha(1-\alpha) & \mu+(1-\alpha)^{2}
\end{array}\right\}, \quad C(x, t)=\left\{\begin{array}{l}
U(x, t) \\
V(x, t)
\end{array}\right\} .
$$

Here $\kappa$ and $\mu$ are non-dimensional constants:

$$
\kappa=\rho_{\mathrm{s}}^{0} / \rho_{0}, \quad \mu=c_{\mathrm{s}} / K .
$$

Table 1

Material constants

Table 2

Non-dimensional constants for typical fuel elements

\begin{tabular}{lc}
\hline $\begin{array}{l}\text { Non-dimensional } \\
\text { quantity }\end{array}$ & Value \\
\hline$\alpha$ & 0.552 \\
$\bar{\rho}$ & 0.29 \\
$\kappa$ & 10.67 \\
$\mu$ & 0.1 \\
\hline
\end{tabular}


The whole system now is determined by the four non-dimensional constants $\alpha, \bar{\rho}, \kappa$ and $\mu ; \bar{\rho}$ is a function of $\alpha[2,3]$ and values for typical fuel elements are given in table $2[5]$.

A special case occurs when the spacer's stiffness ( $c_{\mathrm{s}}$ resp. $\mu$ ) is assumed to be zero. Then the two equations in (4) are linearly dependent, and one can see by direct inserting that

$$
U(x, t)=e V(x, t)
$$

with

$$
\begin{aligned}
& e=-\left\{\alpha(1-\alpha) \rho_{\mathrm{eff}} / \rho_{0}+\bar{\rho}\right\} /\left(\alpha^{2} \rho_{\mathrm{eff}} / \rho_{0}-(\alpha+\bar{\rho})\right), \\
& \rho_{\mathrm{eff}}=\frac{(\alpha+\bar{\rho})(1-\alpha) \kappa+\alpha \bar{\rho}}{\alpha(1-\alpha)[\alpha \kappa+(1-\alpha)]+\bar{\rho}} \rho_{0} .
\end{aligned}
$$

This system then reduces to one equation for the structure velocity, e.g.:

$$
\ddot{V}-\rho_{0} / \rho_{\text {eff }} \frac{\partial^{2}}{\partial x^{2}} V=0 .
$$

By algebraic manipulation it can be shown that the expression (6) for the slip-ratio is identical with the one given in [2]:

$$
e=\{\kappa \alpha(1-\alpha)+\bar{\rho}\} /\{\alpha(1-\alpha)+\bar{\rho}\} .
$$

It is a difficult task to find a solution for (4) or even for (8) in the case of arbitrary initial- and boundary conditions. But for some special cases which have physical significance this is possible and shall be done later. Before, we discuss an experimental verification of the virtual density parameter $\bar{\rho}$.

\section{Experimental investigation of the virtual density}

One main result of the homogenization theory applied to the reactor core was an expression for the virtual density of the fluid-rod system. The virtual density is determined by the parameter $\bar{\rho}$ which is a function of the fluid volume fraction $\alpha$. In [2] theoretically a value $\bar{\rho}=0.29$ was found (for $\alpha=0.552$ ).

Recently at the KWU in Erlangen some experiments [4] have been performed which allow to evaluate the virtual density in a different way. In these experiments the eigenoscillations of a rod bundle were investigated. In two groups of measurements the oscillation frequencies in air $\left(\omega_{\mathrm{a}}\right)$ and water $\left(\omega_{\mathrm{w}}\right)$ were measured. The ratio $\omega_{\mathrm{a}} / \omega_{\mathrm{w}}$ assumed a value of 1.124 .

In order to describe this experimental situation with the model eqs. (1)-(3) we have to add a term $-r \cdot W$ to the right hand side of (2). This term describes the retracting force due to rod-bending on the rod bundle. The parameter $r$ is undetermined but its value is not needed for the following analysis.

In the case where the bundle moves in air we may assume:

$$
\rho_{0}=0, \quad P=0 .
$$

Then the system (1)-(3) reduces to one equation:

$$
(1-\alpha) \rho_{\mathrm{s}}^{0} \ddot{W}=-r W
$$

and thus

$$
\omega_{\mathrm{a}}^{2}=r \cdot\left[(1-\alpha) \rho_{\mathrm{s}}^{0}\right]^{-1} .
$$

In order to calculate the frequency in water we assume fluid and rods to be incompressible $\left(a=a_{\mathrm{s}}=\infty \rightarrow\right.$ 
$K=\infty)($ table 1$)$. Then (3) gives:

$$
\frac{\mathrm{d}}{\mathrm{d} x}(\alpha U+(1-\alpha) \dot{W})=0, \quad-L \leqslant x \leqslant L .
$$

At the boundaries (fixed walls) we have:

$$
(\alpha U+(1-\alpha) \dot{W})(x= \pm L)=0
$$

and thus by (10) and (11):

$$
\alpha U+(1-\alpha) \dot{W}=0 \rightarrow \dot{U}=-\frac{(1-\alpha)}{\alpha} \ddot{W}, \quad-L \leqslant x \leqslant L .
$$

With (12) it is possible to decouple (1) and (2) to get one equation for $W$ :

$$
\ddot{W}\left[\alpha(1-\alpha)^{2} \rho_{0}+\alpha^{2}(1-\alpha) \rho_{\mathrm{s}}^{0}+\rho_{0} \bar{\rho}\right]=-r \alpha^{2} W
$$

The corresponding eigenfrequency is:

$$
\omega_{\mathrm{w}}^{2}=r \alpha^{2} \cdot\left[\alpha(1-\alpha)^{2} \rho_{0}+\alpha^{2}(1-\alpha) \rho_{\mathrm{s}}^{0}+\rho_{0} \bar{\rho}\right]^{-1} .
$$

Finally, we get from (9) and (13)

$$
\bar{\rho}=\kappa(1-\alpha) \alpha^{2}\left[\omega_{\mathrm{w}}^{2} / \omega_{\mathrm{a}}^{2}-1\right]-\alpha(1-\alpha)^{2}
$$

and, equivalently,

$$
\omega_{\mathrm{w}}^{2} / \omega_{\mathrm{a}}^{2}=1+[\bar{\rho}+\alpha(1-\alpha)] /\left[\kappa \alpha^{2}(1-\alpha)\right] .
$$

Taking the values as given in table 2 , i.e. with the theoretical value of $\bar{\rho}$, we compute

$$
\omega_{\mathrm{a}} / \omega_{\mathrm{w}}=1.130 \text {. }
$$

This value differs from the experimental one by $0.5 \%$. On the other hand, we can also compute the appropriate value of $\bar{\rho}$ from the experimental result and obtain

$$
\bar{\rho}=0.271
$$

The difference of the two values of $\bar{\rho}$ is $7 \%$.

We think that these aggreements give a good justification for the homogenization theory approach to this problem. A similar homogenization theory has been developed by Planchard [6]. His result for the effective speed of sound, eq. (1.1) in [6], aggrees well with the result obtained by us for $(1-\alpha) \ll 1$, see $[2$, eq. $(140 \mathrm{a}, 142)]$. However, Planchard's equation (4.12) for the resultant eigenfrequency differs from the above result, eq. (13). In our notation, Planchard's result reads

$$
\omega_{\mathrm{w}}^{2}=r \cdot\left[(1-\alpha)\left(\rho_{\mathrm{s}}^{0}+\rho_{\mathrm{add}}\right)\right]^{-1}, \quad \rho_{\mathrm{add}}:=4 \rho_{0} \frac{1-\alpha}{\alpha} a^{2} / a_{\mathrm{eff}}^{2} .
$$

Taking Planchard's result (1.1),

$$
a^{2} / a_{\text {eff }}^{2}=2-\alpha \text { for }(1-\alpha) \ll 1,
$$

then one gets

$$
\omega_{\mathrm{w}}^{2}=r \cdot\left[(1-\alpha)\left(\rho_{\mathrm{s}}^{0}+\rho_{0} 4(1-\alpha)(2-\alpha) / \alpha\right)\right]^{-1} .
$$

For $(1-\alpha) \ll 1$ we have found that

$$
\bar{\rho} \sim \alpha(1-\alpha),
$$


so that (13) can be written as

$$
\omega_{\mathrm{w}}^{2}=r \cdot\left[(1-\alpha)\left(\rho_{\mathrm{s}}^{0}+\rho_{0}(2-\alpha) / \alpha\right)\right]^{-1} .
$$

Thus, Planchard's result differs from ours. Now, we compare with the experiment. From Planchard's theory we get

$$
\omega_{\mathrm{a}} / \omega_{\mathrm{w}}=[1+4(1-\alpha)(2-\alpha) /(\alpha \kappa)]^{1 / 2},
$$

which gives a value of 1.200 for the experiment of Wehling et al. [4]. This value differs far more $(6.8 \%$ instead of $0.5 \%$ ) from the experimental result than our result.

\section{Analytical solutions for special initial- and boundary conditions}

\subsection{Periodically varying wall velocities}

A solution of (4) (we first discuss the case $\mu>0$ ) is easily derived when we prescribe periodically varying velocity fields at the boundaries:

$$
U(x= \pm 1, t)=V(x= \pm 1, t)=\cos (2 \pi \omega t) .
$$

After many cycles of oscillations the influence of the initial conditions may be neglected. Then, we get a solution of (4) by the ansatz:

$$
C(x, t)=c(x) \cos (2 \pi \omega t),
$$

where $c(x)$ is a vector function

$$
c(x)=\left\{\begin{array}{l}
u(x) \\
v(x)
\end{array}\right\}
$$

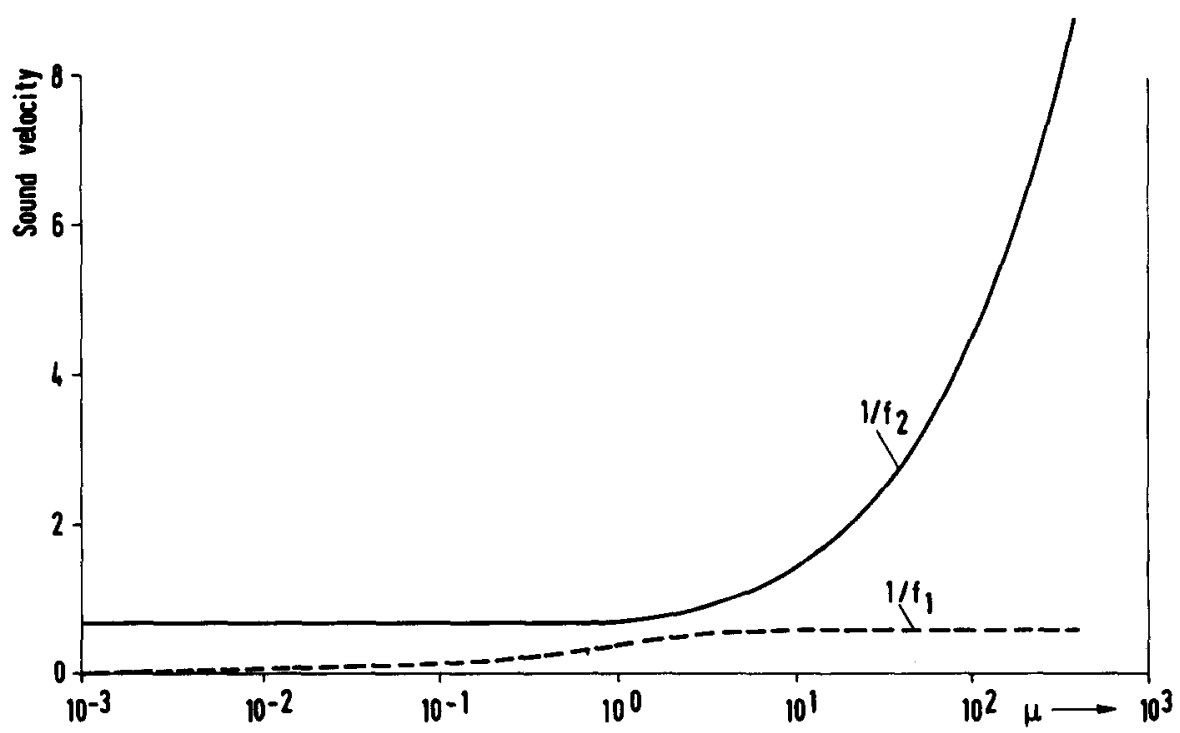

Fig. 1. Non-dimensional sound velocities as function of the spacer's stiffness. 
Substituting (16) into (4) gives a system of two ordinary differential equations for $u$ and $v$ :

$$
\underline{B} \frac{\mathrm{d}^{2}}{\mathrm{~d} x^{2}}\left\{\begin{array}{l}
u \\
v
\end{array}\right\}+4 \pi^{2} \omega \underline{A}\left\{\begin{array}{l}
u \\
v
\end{array}\right\}=0,
$$

with the boundary conditions

$$
u(x= \pm 1)=v(x= \pm 1)=1 .
$$

The system (17) is symmetric and positive definite, therefore its eigenvalues $2 \pi \delta_{i}(i=1,2)$ are positive. These eigenvalues have the meaning of inverse spatial wave lengths. $\delta_{i}=\delta_{i}(\omega)$ is determined by

$$
\operatorname{det}\left(\omega^{2} \underline{A}-\delta_{i}^{2} \underline{B}\right)=0 .
$$

If (19) is fulfilled we have a unique $e_{i}$ with

$$
\left(\omega^{2} \underline{A}-\delta_{i}^{2} \underline{B}\right) E_{i}=0, \quad E_{i}=\left\{\begin{array}{c}
e_{i} \\
1
\end{array}\right\}, \quad(i=1,2) .
$$

Furthermore, we see that the ratio

$$
f_{i}=\delta_{i} / \omega, \quad(i=1,2)
$$

is real and independent of the input value of $\omega$.

By a simple but long algebraic calculation one obtains the following expressions for $e_{i}$ and $f_{i}$ :

$$
\begin{aligned}
f_{1}= & \left\{\frac{\alpha^{2}(1-\alpha) \kappa+\mu(\alpha+\bar{\rho})+\bar{\rho}+\alpha(1-\alpha)^{2}}{2 \alpha^{2} \mu}\right. \\
& \left.\times\left\{1+\left\{1-4 \frac{\alpha^{2} \mu[(\alpha+\bar{\rho})(1-\alpha) \kappa+\alpha \bar{\rho}]}{\left[\alpha^{2}(1-\alpha) \kappa+\mu(\alpha+\bar{\rho})+\bar{\rho}+\alpha(1-\alpha)^{2}\right]^{2}}\right\}^{i / 2}\right\}\right\}^{1 / 2}, \\
f_{2}= & \left.\left.\left.\left\{\frac{\alpha^{2}(1-\alpha) \kappa+\mu(\alpha+\bar{\rho})+\bar{\rho}+\alpha(1-\alpha)^{2}}{2 \alpha^{2} \mu}\right\}^{\alpha^{2} \mu[(\alpha+\bar{\rho})(1-\alpha) \kappa+\alpha \bar{\rho}]}\right\}^{1 / 2}\right\}\right\}^{1 / 2}, \\
& \times\left\{1-\left\{1-4 \frac{\left.\alpha^{2}(1-\alpha) \kappa+\mu(\alpha+\bar{\rho})+\bar{\rho}+\alpha(1-\alpha)^{2}\right]^{2}}{\left[{ }^{2}\right.}\right\}^{2},\right. \\
e_{i}= & \left\{\alpha(1-\alpha) f_{i}^{2}+\bar{\rho}\right\} /\left\{-\alpha^{2} f_{i}^{2}+(\alpha+\bar{\rho})\right\}, \quad(i=1,2) .
\end{aligned}
$$

The values $1 / f_{i}$ correspond to the speed of pressure waves travelling in the fluid-rod mixture. The variation of these speeds with the stiffness parameter $\mu$ can be seen in fig. 1 . The asymptotic values of $1 / f_{i}$ in the cases of very small and very large stiffness are:

(a) For $\mu \ll 1$ :

$$
\begin{aligned}
& f_{1}=\left\{\frac{\alpha^{2}(1-\alpha) \kappa+\bar{\rho}+\alpha(1-\alpha)^{2}}{\alpha^{2} \mu}\right\}^{1 / 2}+O\left(\mu^{1 / 2}\right), \\
& f_{2}=\left[\rho_{\mathrm{eff}} / \rho_{0}\right]^{1 / 2}+O\left(\mu^{1 / 2}\right) .
\end{aligned}
$$


(b) For $\mu \gg 1$ :

$$
\begin{aligned}
& f_{1}=\left\{(\alpha+\rho) / \alpha^{2}\right\}^{1 / 2}+O\left(\mu^{-1 / 2}\right), \\
& f_{2}=O\left(\mu^{-1 / 2}\right) .
\end{aligned}
$$

By (19), (20) and (21) the solution of (17) is

$$
\begin{aligned}
& u(x)=d_{1} e_{1} \cos \left(2 \pi f_{1} \omega x\right)+d_{2} e_{2} \cos \left(2 \pi f_{2} \omega x\right)+d_{3} e_{1} \sin \left(2 \pi f_{1} \omega x\right)+d_{4} e_{2} \sin \left(2 \pi f_{2} \omega x\right), \\
& v(x)=d_{1} \cos \left(2 \pi f_{1} \omega x\right)+d_{2} \cos \left(2 \pi f_{2} \omega x\right)+d_{3} \sin \left(2 \pi f_{3} \omega x\right)+d_{4} \sin \left(2 \pi f_{2} \omega x\right) .
\end{aligned}
$$

The coefficients $d_{i}(i=1, \ldots, 4)$ must be determined from the boundary conditions (18). From (29) and (18) one gets the system of linear equations:

$$
\underline{L} \boldsymbol{D}=\boldsymbol{I},
$$

with

$$
\begin{aligned}
\underline{\boldsymbol{L}} & =\left[\begin{array}{llll}
e_{1} \cos \left(2 \pi f_{1} \omega\right) & e_{2} \cos \left(2 \pi f_{2} \omega\right) & e_{1} \sin \left(2 \pi f_{1} \omega\right) & e_{2} \sin \left(2 \pi f_{2} \omega\right) \\
e_{1} \cos \left(2 \pi f_{1} \omega\right) & e_{2} \cos \left(2 \pi f_{2} \omega\right) & -e_{1} \sin \left(2 \pi f_{1} \omega\right) & -e_{2} \sin \left(2 \pi f_{2} \omega\right) \\
\cos \left(2 \pi f_{1} \omega\right) & \cos \left(2 \pi f_{2} \omega\right) & \sin \left(2 \pi f_{1} \omega\right) & \sin \left(2 \pi f_{2} \omega\right) \\
\cos \left(2 \pi f_{1} \omega\right) & \cos \left(2 \pi f_{2} \omega\right) & -\sin \left(2 \pi f_{1} \omega\right) & -\sin \left(2 \pi f_{2} \omega\right)
\end{array}\right], \\
\boldsymbol{D} & =\left(d_{1}, d_{2}, d_{3}, d_{4}\right)^{\mathrm{T}}, \\
& \boldsymbol{I}=(1,1,1,1)^{\mathrm{T}} .
\end{aligned}
$$

A unique solution of (30) and thus of (17), (18) exists if and only if $\operatorname{det}(\underline{L}) \neq 0$, where

$$
\operatorname{det}(\underline{L})=-\left(e_{1}-e_{2}\right)^{2} \sin \left(4 \pi f_{1} \omega\right) \sin \left(4 \pi f_{2} \omega\right)
$$

The case $\operatorname{det}(\underline{L})=0$ corresponds to the case of resonance and is discussed in the next section.

These calculations are valid for the case $\mu>0$. The case $\mu=0$ is much simpler because one only has to solve one equation (8) instead of a system. In order to fulfill the continuity equation at the walls, we prescribe as boundary condition:

$$
\alpha U(x= \pm 1, t)+(1-\alpha) V(x= \pm 1, t)=\cos (2 \pi \omega t) ;
$$

which implies by (5):

$$
V(x= \pm 1, t)=[\alpha e+(1-\alpha)]^{-1} \cos (2 \pi \omega t) .
$$

Now the solution process is the same as before and results in

$$
\begin{aligned}
& V(x, t)=[(\alpha e+(1-\alpha)) \cos (2 \pi f \omega)]^{-1} \cos (2 \pi f \omega x) \cos (2 \pi \omega t), \\
& U(x, t)=e V(x, t),
\end{aligned}
$$

with

$$
f=\left(\rho_{\text {eff }} / \rho_{0}\right)^{1 / 2}
$$

A comparison of (25), (26) with (34) shows the influence of a positive spacer's stiffness. The wavelength of the 'acoustic' waves $(1 / \omega f)$ which appears for $\mu=0$ is considerably enlarged in the general case $\mu>0$. At the same time this oscillation is superposed by a second one due to relative motion of neighbouring rods (fig. 2). The wavelength $1 / f_{1} \omega$ of this additional oscillation tends to zero for $\mu \rightarrow 0$. For $\mu \leqslant 0.1$ this wavelength is less then $20 \%$ of the length of the acoustic waves. So the influence of the spacers is small or even negligible for $\mu \leqslant 0.1$, at least to a first approximation. 


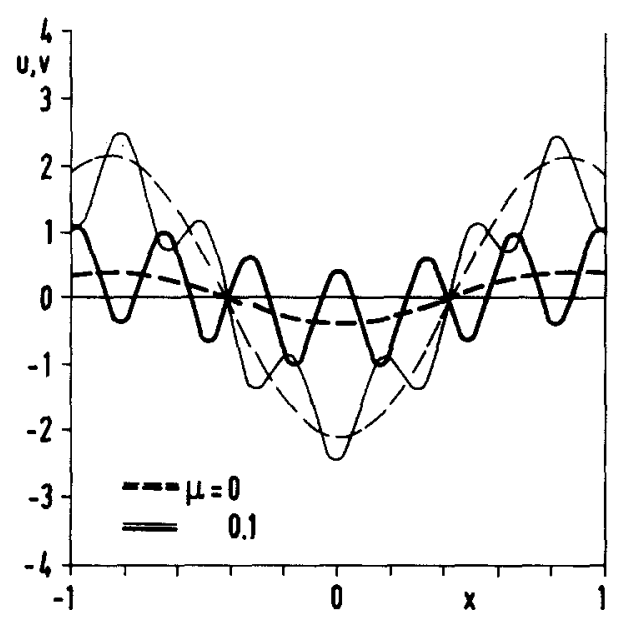

Fig. 2. Velocity distribution in the case of periodic wall oscillation with a non-dimensional frequency $\omega=6$. Thick curves: rod velocity $v$ : thin curves: fluid velocity $u$.

\subsection{Eigenfrequencies and eigenoscillations}

Eigenoscillations of the fluid-rod continuum are solutions of (4) which have the form (16):

$$
C(x, t)=c(x) \cos (2 \pi \omega t) \text {. }
$$

Here we search for eigenoscillations which satisfy the boundary values:

$$
C(x= \pm 1, t)=0, \text { for } t>0 .
$$

In order to fulfill (35) we have to solve, instead of (30), the equation

$$
\underline{L} \boldsymbol{D}=(0,0,0,0)^{\mathrm{T}} \text {. }
$$

This is possible only if $\operatorname{det}(\boldsymbol{L})=0$. Such a solution exists only for some discrete values of $\omega$, the eigenfrequencies of the system. By (31) we see that in the case $\mu>0$ there are two series of eigenfrequencies

$$
\omega_{i, n}=n / 4 f_{i}, \quad(n=1,2,3, \ldots ; i=1,2) .
$$

If $\omega_{i, n}$ is an eigenfrequency, a solution $\boldsymbol{D}_{i, n}$ of (36) exists, unique up to a constant factor.

One can verify that

$$
\begin{array}{ll}
\boldsymbol{D}_{i, n}=\left(\delta_{1, i}, \delta_{2, i}, 0,0\right)^{\mathrm{T}} & \text { if } n=1,3,5, \ldots ; i=1,2, \\
\boldsymbol{D}_{i, n}=\left(0,0, \delta_{1, i}, \delta_{2, i}\right)^{\mathrm{T}} & \text { if } n=2,4,6, \ldots ; i=1,2,
\end{array}
$$

and thus one obtains

$$
\begin{array}{ll}
\boldsymbol{c}_{i, n}(x)=E_{i} \cos \left(\frac{1}{2} n \pi x\right) & \text { if } n=1,3,5, \ldots ; i=1,2 \\
\boldsymbol{c}_{i, n}(x)=E_{i} \sin \left(\frac{1}{2} n \pi x\right) & \text { if } n=2,4,6, \ldots ; i=1,2,
\end{array}
$$

as eigensolutions of (17).

In the case of zero spacer stiffness there exists only one series of eigenfrequencies

$$
\omega_{n}=n / 4 f, \quad(n=1,2,3, \ldots),
$$


with the corresponding eigenoscillations:

$$
\begin{array}{ll}
V_{n}(x, t)=\cos \left(\frac{1}{2} n \pi x\right) \cos \left(2 \pi \omega_{n} t\right) & \text { if } n=1,3,5, \ldots, \\
V_{n}(x, t)=\sin \left(\frac{1}{2} n \pi x\right) \cos \left(2 \pi \omega_{n} t\right) & \text { if } n=2,4,6, \ldots
\end{array}
$$

The influence of the spacer springs lies in the additional series of eigenfrequencies $\omega_{1, n}(n=1,2)$. For moderate stiffness parameters $(\mu \leqslant 0.1)$ the basic frequency $\omega_{2,1}$ of this second series differs little from the frequency $\omega_{1}$ which appears in the case $\mu=0$ (table 3 ). Typical dimensional values are $L=2.155 \mathrm{~m}$, $\tau=1.643 \mathrm{~ms}$ and thus $\omega_{2,1} / \tau=101.35 \mathrm{~Hz}$ for $\mu>0$. If $\mu$ tends to zero one sees from (25), (26) that $\omega_{1, n} \rightarrow 0, \omega_{2, n} \rightarrow \omega_{n}$.

\subsection{Linearly increasing wall accelerations}

A lack of the case considered in the two preceding sections is the independence on initial conditions. So only a 'steady state' was calculated, the velocity distribution after a long time of periodical acceleration.

In order to get a solution with more physical relevance we now consider the case where fluid and rods are initially at rest and the wall acceleration is linearly increasing.

$$
\begin{aligned}
& \underline{A} \frac{\partial^{2}}{\partial t^{2}} C+\underline{B} \frac{\partial^{2}}{\partial x^{2}} C=0, \\
& C(x, 0)=(0,0)^{\mathrm{T}}, \quad \text { for }-1 \leqslant x \leqslant 1, \\
& C(x= \pm 1, t)=\frac{1}{2} t^{2}(1,1)^{\mathrm{T}}, \quad \text { for } t>0 .
\end{aligned}
$$

Perhaps even more interesting would be the case of constant wall accelerations starting at $t=0$. However, this would cause solutions with shocks for which we do not see analytical solutions.

By a simple transformation the inhomogeneity in the system (4), (42), (43) is moved from the boundary condition (43) to the initial condition (42): if $\tilde{C}$ is the solution of (4) with

$$
\begin{aligned}
& \tilde{C}(x, 0) \equiv \tilde{c}(x)=\frac{1}{2}\left(x^{2}-1\right) W, \text { for }-1 \leqslant x \leqslant 1, \\
& \tilde{C}(x= \pm 1, t)=(0,0)^{\mathrm{T}}, \text { for } t>0, \\
& \boldsymbol{W}=\left(W_{1}, W_{2}\right)^{\mathrm{T}} \text { and } W_{1}=\left\{(1-\alpha)^{2}(\kappa-1)\right\} / \mu \alpha-(1 / \alpha), \quad W_{2}=-((1-\alpha)(\kappa-1)) / \mu,
\end{aligned}
$$

then

$$
C(x, t)=\tilde{C}(x, t)+\frac{1}{2} t^{2}(1,1)^{\mathrm{T}}-\frac{1}{2}\left(x^{2}-1\right) W
$$

is the solution of (4), (42), (43).

The system (4), (42a), (43a) can be solved by the method of Fourier. The idea is simply to make a

Table 3

Basic nondimensional eigenfrequencies

\begin{tabular}{lll}
\hline$\mu$ & $\omega_{1,1}$ & $\omega_{2,1}$ \\
\hline 0 & - & 0.1666 \\
$10^{-7}$ & $3.20410^{-5}$ & 0.1666 \\
$10^{-4}$ & $1.01210^{-3}$ & 0.1666 \\
$10^{-1}$ & $3.17010^{-2}$ & 0.1667 \\
1 & $9.54610^{-2}$ & 0.1766 \\
\hline
\end{tabular}


Fourier expansion of the initial function $\tilde{\boldsymbol{c}}(x)$ in the eigenfunctions $\boldsymbol{c}_{i, n}(39)$ :

$$
c(x)=\sum_{n=1}^{\infty} \sum_{i=1}^{2} \alpha_{i, n} c_{i, n}(x) .
$$

By (16) and (35)

$$
\tilde{C}(x, t)=\sum_{n=1}^{\infty} \sum_{i=1}^{2} \alpha_{i, n} c_{i, n}(x) \cos \left(2 \pi \omega_{i, n} t\right)
$$

is the desired solution.

The system (17) is symmetric and positive definite, the eigenfunctions $c_{i, n}$ therefore are orthogonal in the sense that

$$
\int\left(\underline{B}_{i, n}(x) \cdot c_{j, m}(x)\right) \mathrm{d} x=r_{i, n} \delta_{i, j} \delta_{n, m},
$$

and so with (39), (42a) and (44) one gets

$$
\begin{aligned}
& \alpha_{i, n}=\frac{\left(\underline{B} \boldsymbol{W} \cdot \boldsymbol{E}_{i}\right) \int 0.5\left(x^{2}-1\right) \sin \left(\frac{1}{2} n \pi x\right) \mathrm{d} x}{\left(\underline{B}_{i} \cdot \boldsymbol{E}_{i}\right) \int \sin ^{2}\left(\frac{1}{2} n \pi x\right) \mathrm{d} x}=0, \quad \text { for } n=2,4,6, \ldots ; i=1,2, \\
& \boldsymbol{\alpha}_{i, n}=\frac{\left(\underline{B} \boldsymbol{W} \cdot \boldsymbol{E}_{i}\right) \int 0.5\left(x^{2}-1\right) \cos \left(\frac{1}{2} n \pi x\right) \mathrm{d} x}{\left(\underline{\boldsymbol{B}}_{i} \cdot \boldsymbol{E}_{i}\right) \int \cos ^{2}\left(\frac{1}{2} n \pi x\right) \mathrm{d} x}=\quad \begin{array}{l}
(-1)^{(n+1) / 2} \frac{16}{(n \pi)^{3}} \frac{\left(\underline{\boldsymbol{B}} \boldsymbol{W} \cdot \boldsymbol{E}_{i}\right)}{\left(\underline{\boldsymbol{B}}_{i} \cdot \boldsymbol{E}_{i}\right)} \\
\text { for } n=1,3,5, \ldots ; i=1,2 .
\end{array}
\end{aligned}
$$

Here $(\boldsymbol{A} \cdot \boldsymbol{B})$ denotes the scalar product of two vectors $\boldsymbol{A}$ and $\boldsymbol{B}$, all integrals go from -1 to 1 .
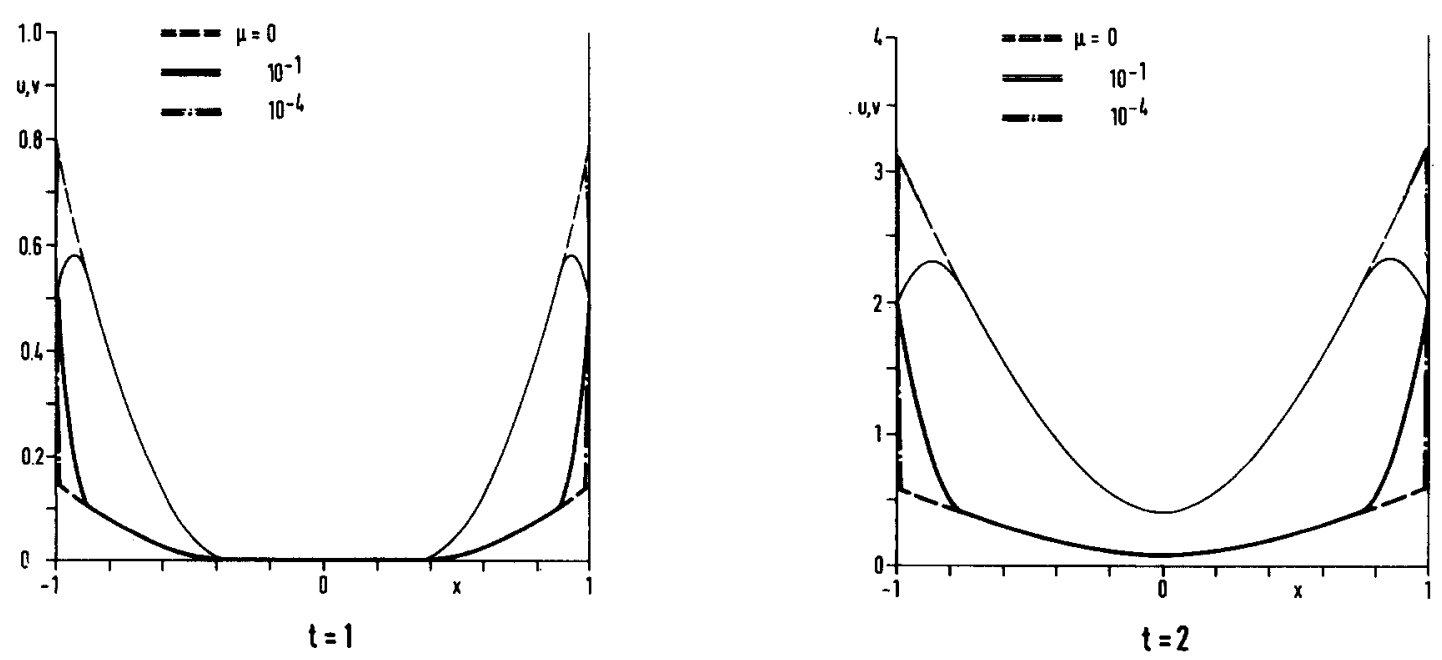

Fig. 3. Case of linearly increasing wall acceleration. Velocity distribution after a nondimensional time $t=1$ for different stiffness parameters $\mu$. Thick curves: rod velocity $v$; thin curves: fluid velocity $u$.

Fig. 4. Same as fig. 3 for $t=2$. 

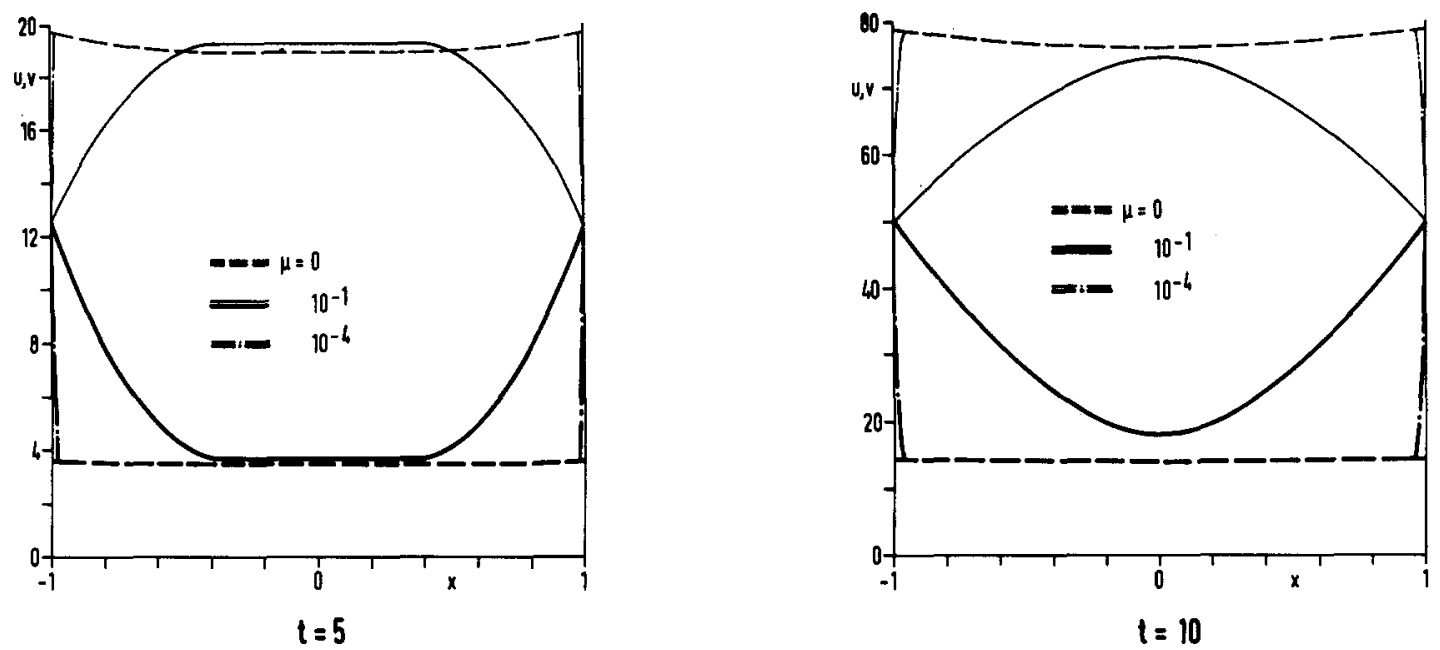

Fig. 5. Same as fig. 3 for $t=5$.

Fig. 6. Same as fig. 3 for $t=10$.

Again, the case $\mu=0$ is simpler,

$$
\tilde{V}(x, t)=\sum_{n=1}^{\infty} \alpha_{n} \cos \left((2 n-1) \frac{1}{2} \pi x\right) \cos \left(2 \pi \omega_{n} t\right),
$$

with

$$
\alpha_{n}=(-1)^{n} 16\left[(\alpha e+(1-\alpha))((2 n-1) \pi)^{3}\right]^{-1} \rho_{\mathrm{eff}} / \rho_{0}, \quad n=1,2,3, \ldots,
$$

and the solution of (8) is

$$
\begin{aligned}
& V(x, t)=\tilde{V}(x, t)+\frac{1}{2}[\alpha e+(1-\alpha)]^{-1} t^{2}-\frac{1}{2}[\alpha e+(1-\alpha)]^{-1} \rho_{\mathrm{eff}} / \rho_{0}\left(x^{2}-1\right), \\
& U(x, t)=e V(x, t)
\end{aligned}
$$

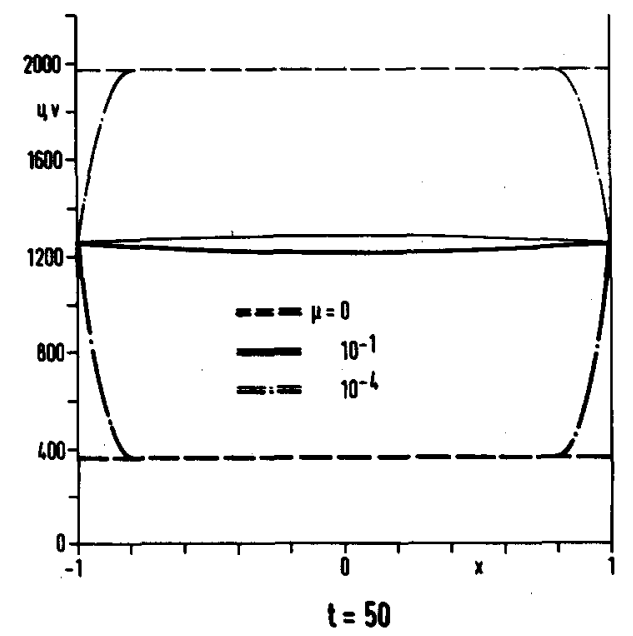

Fig. 7. Same as fig. 3 for $t=50$. 
Figs. 3-7 show the velocity distributions for different spacer's stiffnesses and different times. One can see that in the case of very small stiffness parameters $(\mu \ll 1)$ the influence of the spacer springs is negligible, except for a relatively small boundary layer. This is valid for a long time period.

If the springs have a stiffness of order $\mu=0.1$ (as it was measured for actual rod bundles) their influence is larger. Only for smaH times $(t \leqslant 5)$ this influence is restricted to a boundary layer adjacent to the walls. For later times the slip velocity in the whole region is highly overestimated when the spacer springs are neglected. This shows us the limitations of a core model where stiffness forces are not included.

\section{Effective density of the fluid-rod mixture}

With the solutions derived in the preceeding paragraph it is easy to calculate the dynamic effective density of the fluid-rod mixture. Because of the slip motion between the two phases this effective mass per volume normally is different from the mass-averaged one. The effective density $\rho_{\text {eff }}$ is defined by

$$
\left[\alpha \rho_{0} \dot{u}+(1-\dot{\alpha}) \rho_{\mathrm{s}}^{0} \dot{v}\right]=\rho_{\mathrm{eff}}[\alpha \dot{u}+(1-\alpha) \dot{v}]
$$

where $\dot{u} \equiv \dot{u}(t), \dot{v} \equiv \dot{v}(t)$ are the average accelerations of fluid and rods:

$$
\dot{u}(t)=\frac{1}{2} \int \dot{U}(x, t) \mathrm{d} x, \quad \dot{v}(t)=\frac{1}{2} \int \dot{V}(x, t) \mathrm{d} x .
$$

Thus, in the case of periodically varying wall velocities and positive spacer's stiffness one calculates

$$
\rho_{\text {eff }}=\rho_{0} \frac{\left(d_{1} / f_{1}\right)\left[\alpha e_{1}+(1-\alpha) \kappa\right] \sin \left(2 \pi f_{1} \omega\right)+\left(d_{2} / f_{2}\right)\left[\alpha e_{2}+(1-\alpha) \kappa\right] \sin \left(2 \pi f_{2} \omega\right)}{\left(d_{1} / f_{1}\right)\left[\alpha e_{1}+(1-\alpha)\right] \sin \left(2 \pi f_{1} \omega\right)+\left(d_{2} / f_{2}\right)\left[\alpha e_{2}+(1-\alpha)\right] \sin \left(2 \pi f_{2} \omega\right)},
$$

and for $\mu=0$

$$
\rho_{\text {eff }}=\rho_{0} f^{2} \text {. }
$$

Subsequently we normalize the effective density with the averaged density

$$
\rho_{\max }=\alpha \rho_{0}+(1-\alpha) \rho_{s}^{0} .
$$

In the case $\mu=0$ (51) shows that for a typical core the dynamically effective mass is only $42 \%$ of the physical mass (52), a result which was earlier derived in [7].

Fig. 8 and fig. 9 show the normalized effective density as function of the oscillation frequency $\omega$ and
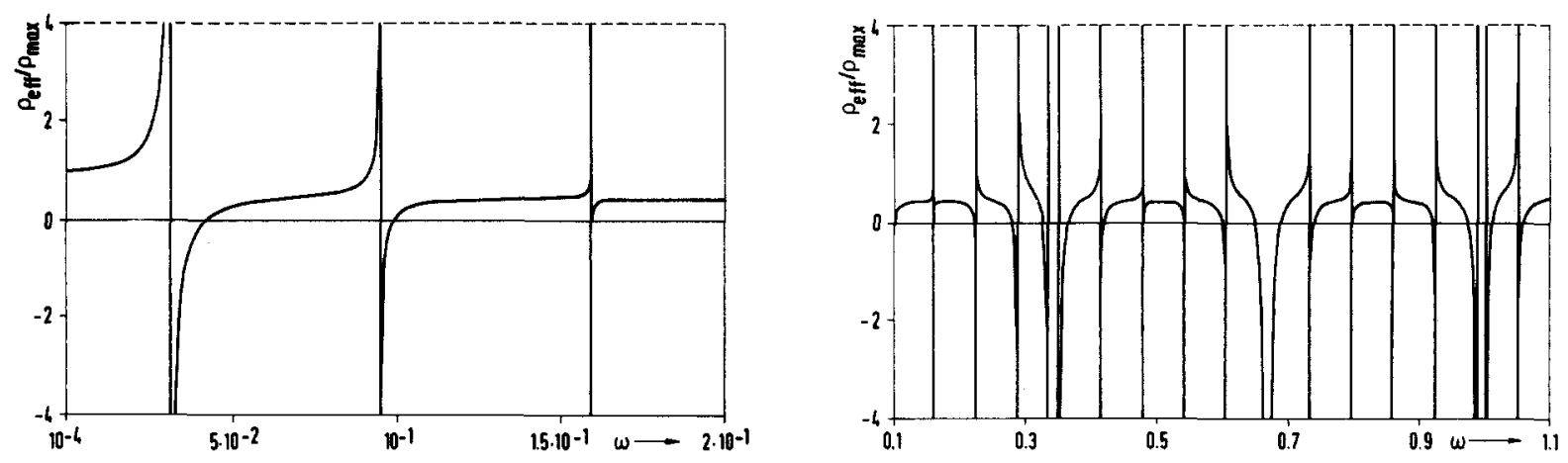

Fig. 8. Case of periodic wall accelerations. Normalized effective density as function of the non-dimensional oscillation frequency. stiffness parameter $\mu=0.1$ fixed. The density curve is cut off at the values 4 and -4 .

Fig. 9. Same as fig. 8 for a larger frequency interval. 


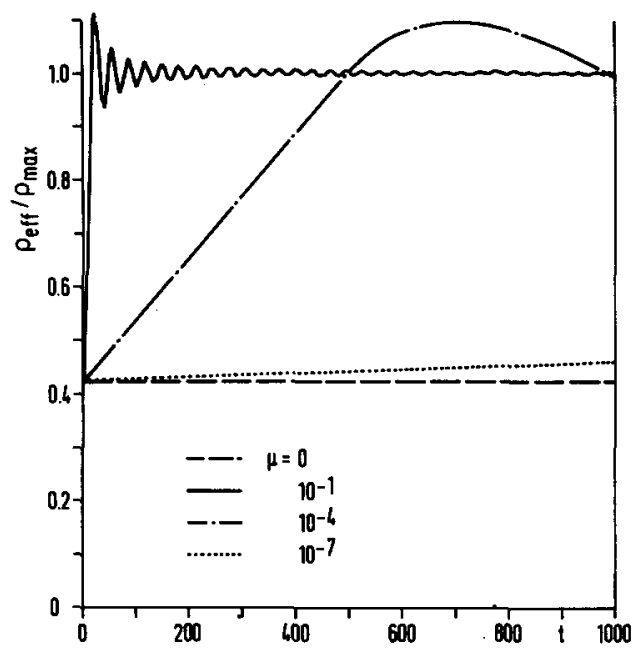

Fig. 10. Case of linearly increasing wall acceleration. Effective density as a function of time for different stiffness parameters.

$\mu$-0.1 fixed. For $\omega \rightarrow 0$ we see that $\rho_{\text {eff }} \rightarrow \rho_{\max }$ (fig. 8), this result is valid for arbitrary $\mu$. Furthermore, one can derive from (50) that $\rho_{\text {eff }} \rightarrow \rho_{\max }$ if $\omega$ is fixed and $\mu \rightarrow \infty$.

If $\omega$ is equal to an eigenfrequency of the continuum, the value of the effective density becomes undefined because no solution of (30) exists. Fig. 9 shows the behaviour of the density curve in the neighbourhood of an eigenfrequency. If $\omega_{i, n}$ is an odd-numbered value of the first series of frequencies (i.e., $i=1$ and $n=1,3,5, \ldots)$ then $\rho_{\text {eff }}(\omega)$ becomes discontinuous in the neighbourhood of $\omega_{i, n}$. The same occurs if $\omega_{i, n}$ is an even numbered value in the second series (i.e. $i=2, n=2,4,6, \ldots$ ). For $\omega$ far away from any eigenfrequency, $\rho_{\text {eff }}(\omega)$ tends to the value 0.42 which was found previously in the case $\mu=0$.

The case of linearly growing wall acceleration is treated in the same way. With $\mu=0$ the result is identical with the previous one, for all times the effective mass is $42 \%$ of the physical mass. More interesting is again the case $\mu>0$. Now with increasing time the spacers reduce the slip ratio and therefore the effective mass increases (fig. 10). Here one again sees the limitations of a core model without spacer springs. With $\mu=0.1$, already after a nondimensional time of 10 the effective mass is $98 \%$ of the physical mass, instead of $42 \%$ in the case $\mu=0$.

\section{Conclusions}

In this paper a one-dimensional homogenized model for a pressurized water reactor core was investigated. This model is given by a system of partial differential equations for fluid velocity, rod velocity and pressure. For these equations and some special initial and boundary conditions analytical solutions were derived.

The importance of the virtual density in the model equations was shown. It was shown that the theoretically calculated virtual density parameter is in good aggreement with experimental data.

The main result is quantitative information on the influence of the spacer's stiffness. For realistic values of the stiffness-parameter $\left(\mu \equiv c_{\mathrm{s}} / K \leqslant 0.1\right)$ it was found that in some cases the spacers have a minor influence outside a small boundary layer adjacent to the walls. The error made by neglecting stiffness forces becomes larger with increasing times. Nevertheless, for a three-dimensional core model this simplification seems to be appropriate to a first approximation. It will reduce the computational effort for implementing the model in a code for the analysis of pressure loadings during a loss-of-coolant accident. 


\section{References}

[1] U. Schumann, Homogenized equations of motion for rod bundles in fluid with periodic structure, Ing.-Arch. 50 (1981) $203-216$

[2] U. Schumann, Virtual density and speed of sound in a fluid-solid mixture with periodic structure, Internat. J. Multiphase Flow (1981).

[3] U. Schumann and J. Benner, Homogenized model for fluid-structure interaction in a Pressurized Water Reactor core, SMiRT-6, Paris, August 1981, paper B5/7.

[4] H.-J. Wehling, J.D. Kim, A. Nink, G. Stern and H. Stölben, Experimentelle Bestimmung der dynamischen Kennwerte von Brennelementen für Druckwasserreaktoren, SMiRT-6, Paris, August 1981, paper D3/7.

[5] Kraftwerk Union, personal communication, 1981.

[6] J. Planchard, Computation of the acoustic eigenfrequencies of cavities containing a tube bundle, Comput. Meths. Appl. Mech. Engrg. 24 (1980) 125-135.

[7] U. Schumann, Fluid-Struktur-Wechselwirkung des Kernmantels und der Brennstäbe beim Kühlmittelverluststörfall, Atomkernenergie-Kerntechnik 36 (1980) 305-310. 